\title{
STABILITY IMPACT OF SMALL DELAYS IN PROPORTIONAL-DERIVATIVE STATE FEEDBACK ${ }^{1}$
}

\author{
Tomáš Vyhlídal ${ }^{\mathrm{a}}$, Wim Michiels ${ }^{\mathrm{b}}$, Pavel Zítek ${ }^{\mathrm{a}}$ \\ and Paul McGahan ${ }^{\mathrm{a}}$ \\ ${ }^{a}$ Centre for Applied Cybernetics, Dept. of Instrumentation and Control Eng., Fac. \\ of Mechanical Eng., Czech Technical University in Prague \\ e-mail: Tomas.Vyhlidal@fs.cvut.cz,Pavel.Zitek@fs.cvut.cz, \\ Paul.McGahan@fs.cvut.cz \\ ${ }^{\mathrm{b}}$ Dept. of Computer Science, Katholieke Universiteit Leuven. \\ e-mail :Wim.Michiels@cs.kuleuven.be
}

\begin{abstract}
This paper points out specific stability problems of the proportional-derivative state feedback which may result from the presence of otherwise negligible delays in the control loops. It is shown that the stability problems arise from the neutral character of the closed loop system with the state derivative action under small delay perturbations. As the main result, a stability criterion is derived for a safe implementation of the state derivative feedback. Furthermore, a scheme including filtered state derivative feedback is proposed to overcome the destabilizing effects of small feedback delays. Two application examples are presented. From a theoretical point of view, the main contribution of the paper lies in the stability theory of linear neutral equations with dependent delays, subjected to delay perturbations.
\end{abstract}

Key words: state feedback, derivative feedback, neutral system, strong stability, vibration suppression, regenerative chatter

$\overline{1}$ This work was partly done while Wim Michiels was on leave at the Faculty of Mechanical Engineering, Eindhoven University of Technology, The Netherlands. 


\section{Notations}

$\mathbb{C}$

$i$

$\mathbb{N}$

$\mathbb{R}$

$\mathbb{R}^{+}$

$\mathbb{R}_{0}^{+}$

$\Re(\lambda), \Im(\lambda),|\lambda| \quad \lambda \in \mathbb{C}$

$\vec{r} \in \mathbb{R}^{m}, \vec{n} \in \mathbb{N}^{m}, \ldots$

$r_{\sigma}(A)$

$\sigma(A)$

$\operatorname{sign}(x), x \in \mathbb{R}$

$\mathbb{Z}$

$\alpha(A)$

$\|\vec{a}\|, \vec{a} \in \mathbb{R}^{m}$

$\vec{a} \cdot \vec{b}, \vec{a}, \vec{b} \in \mathbb{R}^{m}$

$f(\lambda ; p)$ set of complex numbers

imaginary unit

set of natural numbers, includes zero

set of real numbers

$\{r \in \mathbb{R}: r \geq 0\}$

$\mathbb{R}^{+} \backslash\{0\}$

real part, imaginary part and modulus of $\lambda$

short notation for $\left(r_{1}, \ldots, r_{m}\right),\left(n_{1}, \ldots, n_{m}\right), \ldots$

spectral radius of operator (or matrix) $A$

spectrum of operator (or matrix) $A$

$\operatorname{sign}(x)=\left\{\begin{array}{r}1, x \geq 0 \\ -1, x<0\end{array}\right.$

set of integer numbers

spectral abscissa of operator (or matrix) A,

$\alpha(A):=\sup \{\Re(\lambda): \lambda \in \mathbb{C}$ and $\lambda \in \sigma(A)\}$

Euclidean norm of $\vec{a},\|\vec{a}\|:=\sqrt{\sum_{k=1}^{m} a_{k}^{2}}$

Euclidean inner product of $\vec{a}$ and $\vec{b}, \vec{a} \cdot \vec{b}:=\sum_{k=1}^{m} a_{k} b_{k}$

function $f$, evaluated at argument $\lambda$, that

depends on some parameter $p$, 


\section{Introduction}

The paper deals with the impact on stability of small uncertain delays appearing in the feedback loop of a system controlled by proportional-derivative state feedback. It is well known that small delays systematically originate from the latency effect of implementing the measurements and control actions, occurring, e.g., as a consequence of computational delays, delays arising from AD-DA conversion or communication delays, see Fig. 1. As a rule, such delays are very small compared to the dominant modes of the system, which justifies to neglect them in the feedback design in most applications. However, it is shown in the paper that such delays cannot always be safely neglected if state derivatives are used for feedback, as even arbitrarily small implementation delays may cause instability.

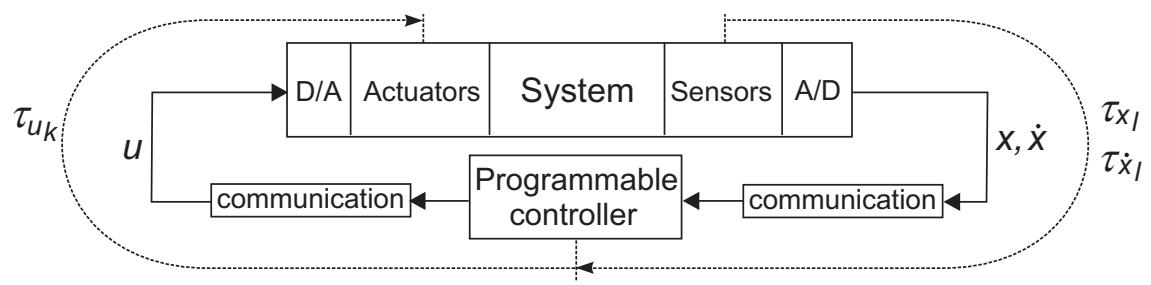

Fig. 1. Small feedback delays in the closed-loop implementation

The delay free system is considered in the form

$$
\dot{x}(t)=A x(t)+B u(t)
$$

where $x \in \mathbb{R}^{n}$ is the vector of state variables, $u \in \mathbb{R}^{n_{u}}$ is the vector of inputs and $A, B$ are constant coefficient matrices of compatible dimension. The proportional-derivative (PD) state controller is given as follows

$$
u(t)=-K_{p} x(t)-K_{d} \dot{x}(t)
$$

where $K_{p} \in \mathbb{R}^{n_{u} \times n}$ and $K_{d} \in \mathbb{R}^{n_{u} \times n}$ are the feedback gain matrices. The closed loop system (1)-(2) is given by

$$
\dot{x}(t)=\left(I+B K_{d}\right)^{-1}\left(A-B K_{p}\right) x(t)
$$

Obviously, the $n$ eigenvalues of the matrix $\left(I+B K_{d}\right)^{-1}\left(A-B K_{p}\right)$ determine the stability and the dynamic behavior.

Consider that delays appear in the feedback paths of the system (1)-(2) as follows: a delay $\tau_{u_{k}}$ on the $k$-th component of input $u, 1 \leq k \leq n_{u}$, a delay $\tau_{\dot{x}_{l}}$ in the measurement of the $l$-th component of $\dot{x}$, and a delay $\tau_{x_{l}}$ in the measurement of the $l$-th component of $x, 1 \leq l \leq n$, as it is shown in Fig. 2, 


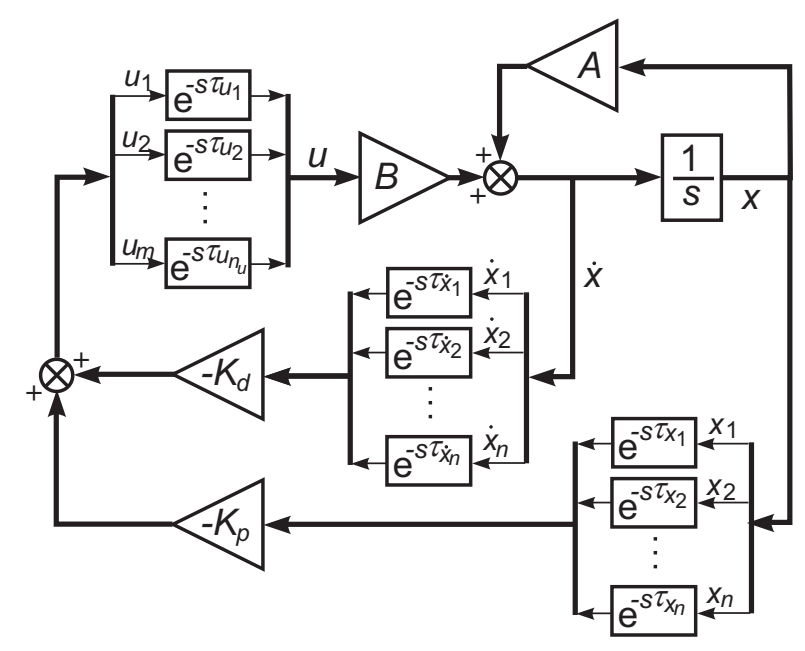

Fig. 2. Scheme of the derivative feedback system with measurement and input delays then the closed-loop system becomes:

$$
\begin{aligned}
& \dot{x}(t)+\sum_{k=1}^{n_{u}} B E_{k} \sum_{l=1}^{n} K_{d} F_{l} \dot{x}\left(t-\tau_{u_{k}}-\tau_{\dot{x}_{l}}\right) \\
& A x(t)-\sum_{k=1}^{n_{u}} B E_{k} \sum_{l=1}^{n} K_{p} F_{l} x\left(t-\tau_{u_{k}}-\tau_{x_{l}}\right),
\end{aligned}
$$

where $E_{k}=\left[e_{i, j}^{k}\right] \in \mathbb{R}^{n_{u} \times n_{u}}$ and $F_{l}=\left[f_{i, j}^{l}\right] \in \mathbb{R}^{n \times n}$ satisfy

$$
e_{i, j}^{k}=\left\{\begin{array}{ll}
1, & i=j=k \\
0, & \text { otherwise }
\end{array}, \quad f_{i, j}^{l}=\left\{\begin{array}{ll}
1, & i=j=l \\
0, & \text { otherwise }
\end{array},\right.\right.
$$

for $k=1, \ldots, n_{u}$ and $l=1, \ldots, n$.

If $K_{d} \neq 0$, then the system (4) is a time-delay system of neutral type, see Hale and Verduyn Lunel (1993). The neutral type of the system induces complications w.r.t. stability issues. For instance, a neutral system may have infinitely many characteristic roots located to the right of the stability boundary (Bellman and Cooke, 1963). Thus a neutral system can be unstable with infinitely many unstable roots. Moreover, the position of the roots may be very sensitive to changes in the delays. It has been shown in Hale and Verduyn Lunel (1993); Avellar and Hale (1980); Hale and Verduyn Lunel (2002), see also Hale and Verduyn Lunel (2001); Michiels, et al. (2002), that even infinitesimal changes in the delays may destabilize neutral systems. The main objective of this paper is to study the induced implementation risk of using state derivative feedback in the control, and to provide adequate solutions if robustness problems appear. From a theoretical point of view, this requires a thorough study of the stability properties of neutral systems with small dependent delays, and their relations with the stability properties of the corresponding delay free systems. 
The structure of the paper is as follows. In section 3 design issues are addressed and the use of PD state controllers is motivated. Since the theory of neutral systems plays a crucial role in investigating the stability of the closed loop system in the presence of small delays, the relevant theoretical results are provided in section 4 . First, conditions are rehearsed under which the stability of a neutral system is robust against small delay perturbations. Next, the transition from a neutral system to a delay free system is studied from a stability point of view. In section 5 a criterion is presented under which the stability of a PD state feedback controlled system is robust against small feedback delays. Furthermore, a practical solution is proposed for enhancing the robustness against feedback delays by using a first order filter in the control loop. The results are illustrated with two application examples in section 6. The first example concerns vibration control of a platform. The second example concerns damping of regenerative chatter in turning processes. The conclusions are presented in section 7 .

\section{State feedback versus state derivative feedback}

The problem of designing a state feedback controller,

$$
u(t)=-K_{p} x(t)
$$

for systems with multiple inputs has been widely studied, see e.g. Kautsky and Nichols (1985); Valášek and Olgac (1995). State feedback is a well established tool for the stabilization and control of systems, and it is widely used in the field of control engineering. The idea of using pure state derivative feedback,

$$
u(t)=-K_{d} \dot{x}(t)
$$

is relatively new, and so far only few applications can be found in the literature. In Abdelaziz and Valášek (2005a, 2004, 2005b) the motivation for using state derivative feedback instead of conventional state feedback is given by the easy implementation in mechanical applications where accelerometers and velocity sensors are used for measuring the system's motion. Thus, the accelerations and velocities are the sensed variables, as opposed to the displacements. Typical applications are in vibration control of mechanical systems (Abdelaziz and Valášek, 2005a, 2004; Guo, Ma and Qiao, 2006). The use of acceleration feedback in vibration suppression problems has also been discussed at length in Olgac, et al. (1997) and Dyke, et al. (1996). The application of state derivative feedback to vibration problems has been discussed from the perspective of robust control in Leitmann and Reithmeier (2003).

Conditions for pole placement by pure state derivative feedback and the relation with pole placement by conventional state feedback are given in Abdelaziz 
and Valášek (2004) as follows (see also Abdelaziz and Valášek (2005c)):

Proposition 3.1 If the pair $(A, B)$ is controllable, then all the characteristic roots of the closed-loop system can be assigned at arbitrary positions in $\mathbb{C} \backslash\{0\}$ using the control law (7) if and only if $\operatorname{det}(A) \neq 0$.

Proposition 3.2 Assume that $\operatorname{det}(A) \neq 0$. If

$$
K_{d}=K_{p}\left(A+B K_{p}\right)^{-1}
$$

then the control law (7) results in the same characteristic roots as the control law (6).

Thus, at the one hand the control law (7) can also be used for stabilization and pole placement, where Proposition 3.2 can be applied for computing the gain. On the other hand the use of pure state derivative feedback for control purposes is limited due to its purely dynamical character. For example, the steady state of the closed loop system is invariant with respect to changes of the feedback gain $K_{d}$. Thus, pure derivative feedback can only be used for the stabilization of the system or for the damping of the dynamic disturbances but not for the regulation to a given set-point.

The control law (2), which corresponds, e.g., to feeding back positions, velocities and accelerations in vibration control applications, combines the positive features of the proportional and derivative control laws discussed above. As it features more parameters than necessary for pole placement, additional degrees of freedom are introduced in the control design, and, unlike pure state derivative feedback, it can still be used for set-point control.

Even though the use of the state derivative control part in (2) may be convenient in applications, due to the presence of delays in the feedback paths, the closed loop system becomes a neutral system of the form (4), and, hence, small delays may affect the stability. This will be investigated in the remainder of the paper.

\section{Stability of neutral type systems}

In order to assess the robustness of the feedback system (1)-(2) against small feedback delays in section 5, the relevant stability theory for neutral systems is developed. More precisely, in this section, stability properties of the general linear neutral equation

$$
\dot{x}(t)+\sum_{k=1}^{p_{1}} H_{k} \dot{x}\left(t-\tau_{k}\right)=A_{0} x(t)+\sum_{k=1}^{p_{2}} A_{k} x\left(t-v_{k}\right),
$$


are discussed, where $x(t) \in \mathbb{R}^{n}$ is the state variable, $\vec{\tau}:=\left(\tau_{1}, \ldots, \tau_{p_{1}}\right) \in\left(\mathbb{R}_{0}^{+}\right)^{p_{1}}$ and $\vec{v}:=\left(v_{1}, \ldots, v_{p_{2}}\right) \in\left(\mathbb{R}_{0}^{+}\right)^{p_{2}}$ are time-delays, and $H_{k}, A_{0}$ and $A_{k}$ are real matrices.

Motivated by the structure of (4), in the study of the dependence of the stability properties on the delay parameters, it is assumed that the delays $\tau_{k}, 1 \leq k \leq p_{1}$, in (8) are not necessarily independent of each other, but are linear functions of $m$ 'independent' parameters $\vec{r}=\left(r_{1}, \ldots, r_{m}\right) \in\left(\mathbb{R}_{0}^{+}\right)^{m}$, as described in the following relation:

$$
\tau_{k}=\vec{\gamma}_{k} \cdot \vec{r}, \quad k=1, \ldots, p_{1},
$$

with $\vec{\gamma}_{k}:=\left(\gamma_{k, 1}, \ldots, \gamma_{k, m}\right) \in \mathbb{N}^{m} \backslash\{\overrightarrow{0}\}$, for $k=1, \ldots, p_{1}$.

It is further assumed that $I+\sum_{k=1}^{p_{1}} H_{k}$ is non-singular, which guarantees that Equation (8) is well posed if all the delays are set to zero.

Based on the monographs (Hale and Verduyn Lunel, 1993; Michiels and Niculescu, 2007), some spectral properties and stability results for (8) are recalled below. The neutral system (8) is exponentially stable if and only if $c_{N}(\vec{r}, \vec{v})<0$, where

$$
c_{N}(\vec{r}, \vec{v})=\sup \left\{\Re(\lambda): \operatorname{det}\left(\Delta_{N}(\lambda ; \vec{r}, \vec{v})\right)=0\right\} .
$$

In this expression the characteristic matrix $\Delta_{N}$ is given by

$$
\Delta_{N}(\lambda ; \vec{r}, \vec{v})=\left(\lambda \Delta_{D}(\lambda ; \vec{r})-A_{0}-\sum_{k=1}^{p_{2}} A_{k} e^{-\lambda v_{k}}\right)
$$

and

$$
\Delta_{D}(\lambda ; \vec{r})=\left(I+\sum_{k=1}^{p_{1}} H_{k} e^{-\lambda \vec{\gamma}_{k} \cdot \vec{r}}\right)
$$

With (8) one can associate the delay-difference equation

$$
x(t)+\sum_{k=1}^{p_{1}} H_{k} x\left(t-\vec{\gamma}_{k} \cdot \vec{r}\right)=0
$$

This equation (11) is exponentially stable if and only if $c_{D}(\vec{r})<0$, where

$$
c_{D}(\vec{r})=\left\{\begin{array}{lr}
-\infty, & \operatorname{det}\left(\Delta_{D}(\lambda ; \vec{r})\right) \neq 0, \forall \lambda \in \mathbb{C}, \\
\sup \left\{\Re(\lambda): \operatorname{det}\left(\Delta_{D}(\lambda ; \vec{r})\right)=0\right\}, & \text { otherwise. }
\end{array}\right.
$$

An important relation in the stability theory of neutral equations is given by:

$$
c_{D}(\vec{r}) \leq c_{N}(\vec{r}, \vec{v}) .
$$


It implies that the exponential stability of (11) is a necessary condition for the exponential stability of (8).

In what follows the solutions of $\operatorname{det}\left(\Delta_{N}(\lambda ; \vec{r}, \vec{v})\right)=0$ are called the characteristic roots of the neutral system (8). Analogously the solutions of $\operatorname{det}\left(\Delta_{D}(\lambda ; \vec{r})\right)=$ 0 are called the characteristic roots of the difference equation (11).

\subsection{Strong exponential stability}

It is well known that the functions $\vec{r} \mapsto c_{D}(\vec{r}),(\vec{r}, \nu) \mapsto c_{N}(\vec{r}, \vec{\nu})$ are not continuous, see e.g. Hale and Verduyn Lunel (1993, 2002); Henry (1974); Michiels and Vyhlídal (2005). As a consequence, from a practical point of view the smallest upper bounds on the real parts of the characteristic roots are to be found, which are 'insensitive' to small delay changes. Such an upper bound $\bar{C}_{D}(\vec{r})$ is defined as follows:

Definition 1 For $\vec{r} \in\left(\mathbb{R}_{0}^{+}\right)^{m}$, let $\bar{C}_{D}(\vec{r}) \in \mathbb{R}$ be defined as

$$
\bar{C}_{D}(\vec{r})=\lim _{\epsilon \rightarrow 0+} c_{\epsilon}(\vec{r})
$$

where

$$
c_{\epsilon}(\vec{r})=\sup \left\{c_{D}(\vec{r}+\delta \vec{r}): \delta \vec{r} \in \mathbb{R}^{m} \text { and }\|\delta \vec{r}\| \leq \epsilon\right\}
$$

Clearly $\bar{C}_{D}(\vec{r}) \geq c_{D}(\vec{r})$, and the inequality can be strict, as shown in Michiels and Vyhlídal (2005) and illustrated later. Further properties of $\bar{C}_{D}(\vec{r})$ are given in the following proposition.

Proposition 2 The following assertions hold:

(1) the function

$$
\vec{r} \in\left(\mathbb{R}_{0}^{+}\right)^{m} \mapsto \bar{C}_{D}(\vec{r})
$$

is continuous;

(2) $\bar{C}_{D}(\vec{r})=c_{D}(\vec{r})$ for rationally independent ${ }^{2} \vec{r}$;

(3) for all $\vec{r}_{1}, \vec{r}_{2} \in\left(\mathbb{R}_{0}^{+}\right)^{m}$

$$
\operatorname{sign}\left(\bar{C}_{D}\left(\vec{r}_{1}\right)\right)=\operatorname{sign}\left(\bar{C}_{D}\left(\vec{r}_{2}\right)\right) ;
$$

(4) the function

$$
(\vec{r}, \vec{v}) \in\left(\mathbb{R}_{0}^{+}\right)^{m} \times\left(\mathbb{R}^{+}\right)^{p_{2}} \mapsto \max \left(\bar{C}_{D}(\vec{r}), c_{N}(\vec{r}, \vec{v})\right)
$$

$\overline{2}$ The $m$ components of $\vec{r}=\left(r_{1}, \ldots, r_{m}\right)$ are rationally independent if and only if $\sum_{k=1}^{m} n_{k} r_{k}=0, n_{k} \in \mathbb{Z}$ implies $n_{k}=0, \forall k=1, \ldots, m$. For instance, two delays $r_{1}$ and $r_{2}$ are rationally independent if their ratio is an irrational number. 
is continuous.

PROOF. Assertions 1) and 2) are direct corollaries of Lemma 2.5 and Theorem 2.2 of Avellar and Hale (1980). The proof of Assertion 3) is due to Proposition 2 of Michiels, et al. (2007) and Assertion 4) is due to Proposition 6 of Michiels, et al. (2007).

Note that by (13) the function (15) is the smallest continuous upper bound on $c_{N}(\vec{r}, \vec{v})$. The property (14) leads us to the following definition:

Definition 3 Let $\Xi:=\operatorname{sign}\left(\bar{C}_{D}(\vec{r})\right), \vec{r} \in\left(\mathbb{R}_{0}^{+}\right)^{m}$.

A consequence of the non-continuity of $c_{D}$ and $c_{N}$ w.r.t. $\vec{r}$ is that arbitrarily small perturbations on the delays may destroy exponential stability. This has lead to the introduction of the concept of strong stability in Hale and Verduyn Lunel (2002): the equation (8) or (11) is strongly exponentially stable if and only if it is exponentially stable and remains so when subjected to small variations in the delays $\vec{r}$ (and $\vec{v}$ ). For instance, the following result holds (Michiels, et al., 2007):

Proposition 4 The necessary and sufficient exponential stability conditions are as follows:

(1) The delay difference equation (11) is strongly exponentially stable if and only if $\Xi<0$.

(2) The neutral equation (8) is strongly exponentially stable if and only if $c_{D}(\vec{r}, \vec{\nu})<0$ and $\Xi<0$.

To conclude, computational formulae for the quantities $\bar{C}_{D}(\vec{r})$ and $\Xi$ are mentioned, which, by Proposition 4 , directly result in strong stability conditions:

Theorem 4.1 Assume that $\operatorname{det} \Delta_{D}(\lambda ; \vec{\tau}) \not \equiv 1$. The following assertions hold:

(1) $\Xi=\operatorname{sign} \log \left(\delta_{0}\right)$, where

$$
\delta_{0}:=\max _{\vec{\theta} \in[0,2 \pi]^{m}} \alpha\left(\sum_{k=1}^{m}-\tilde{H}_{k} e^{-i \theta_{k}}\right)
$$

and the matrices $\tilde{H}_{k}, 1 \leq k \leq m$ are such that

$$
\operatorname{det} \Delta_{D}(\lambda ; \vec{r})=\operatorname{det}\left(I+\sum_{k=1}^{m} \tilde{H}_{k} e^{-\lambda r_{k}}\right)
$$


(2) for all $\vec{r} \in\left(\mathbb{R}_{0}^{+}\right)^{m}, \bar{C}_{D}(\vec{r})$ is the unique zero of the strictly decreasing function

$$
c \in \mathbb{R} \rightarrow f(c ; \vec{r})=\max _{\vec{\theta} \in[0,2 \pi]^{m}} \alpha\left(\sum_{k=1}^{m}-\tilde{H}_{k} e^{-c r_{k}} e^{-i \theta_{k}}\right) ;
$$

(3) if $\delta_{0}>1$, then $\bar{C}_{D}\left(\vec{r}_{0}\right)>0$ for some $\vec{r}_{0} \in\left(\mathbb{R}_{0}^{+}\right)^{m}$.

PROOF. The assertions 1) and 2) are due to Theorem 7 of Michiels, et al. (2007) and the assertion 3) is due to Proposition 3 of Michiels, et al. (2007).

The matrices $\tilde{H}_{k}, 1 \leq k \leq m$, appearing in (16), can be computed by representing the polynomial

$$
p\left(x_{1}, \ldots, x_{m}\right):=\operatorname{det}\left(I+\sum_{k=1}^{p_{1}} H_{k}\left(\Pi_{l=1}^{m} x_{l}^{\gamma_{k} l}\right)\right)
$$

as the determinant of a pencil:

$$
p\left(x_{1}, \ldots, x_{m}\right)=\operatorname{det}\left(I+\sum_{k=1}^{m} \tilde{H}_{k} x_{k}\right) .
$$

This can be done using the techniques described in Michiels, et al. (2007), which are based on an appropriate transformation of the polynomial to a linear fractional representation. In particular cases, this "lifting step", which typically leads to an increase of the dimension in the sense that the dimensions of $\tilde{H}_{k}$ are larger than those of $H_{k}$, can be avoided by putting conditions on the interdependence of the delays. For instance, the following result holds (Michiels, et al., 2007):

Proposition 4.2 If $\operatorname{det} \Delta_{D}(\lambda ; \vec{r}) \not \equiv 0$ and there is a vector $\vec{\beta} \in\left(\mathbb{R}_{0}^{+}\right)^{m}$ such that

$$
\vec{\gamma}_{k} \cdot \vec{\beta}=\vec{\gamma}_{l} \cdot \vec{\beta}, \quad \forall k, l \in\left\{1, \ldots, p_{1}\right\},
$$

then $\Xi=\operatorname{sign} \log \left(\delta_{0}\right)$, where

$$
\delta_{0}:=\max _{\vec{\theta} \in[0,2 \pi]^{m}} \alpha\left(-\sum_{k=1}^{p_{1}} H_{k} e^{-i \vec{\gamma}_{k} \cdot \vec{\theta}}\right) .
$$

\subsection{Transition from a delay free to a neutral system}

In this section, the spectral properties of the system (8) are discussed under the assumption that the delays $\vec{r}$ and $\vec{v}$ are small, and connections with the 
delay-free case are established, where (8) reduces to

$$
\dot{x}(t)=A_{c l} x(t)
$$

with

$$
A_{c l}:=\left(I+\sum_{k=1}^{p_{1}} H_{k}\right)^{-1}\left(A_{0}+\sum_{k=1}^{p_{2}} A_{k}\right)
$$

The next results says, roughly speaking, that for small values of $\vec{r}$ and $\vec{v}$, the system (8) has (at least) $n$ characteristic roots which approximate the eigenvalues of $A_{c l}$. Let us remark that unlike the other results presented in Section 4, which are related to the previous work, the results presented in this subsection have been derived by the authors for this contribution. In order to increase readability, the proofs of propositions are given in the appendix.

Proposition 4.3 Let $\mu_{i} \in \mathbb{C}, 1 \leq i \leq n$, be the eigenvalues of $A_{c l}$, defined by (19). There exist numbers $\hat{\tau}$ and $n$ continuous functions

$$
\hat{\lambda}_{i}:[0, \hat{\tau}]^{m} \times[0, \hat{\tau}]^{p_{2}} \rightarrow \mathbb{C},(\vec{r}, \vec{v}) \mapsto \hat{\lambda}_{i}(\vec{r}, \vec{v}), 1 \leq i \leq n,
$$

which satisfy

$$
\operatorname{det} \Delta_{N}\left(\hat{\lambda}_{i}(\vec{r}, \vec{v}) ; \vec{r}, \vec{v}\right) \equiv 0
$$

and

$$
\lim _{(\vec{r}, \vec{v}) \rightarrow \overrightarrow{0}} \hat{\lambda}_{i}(\vec{r}, \vec{v})=\mu_{i}, \quad 1 \leq i \leq n
$$

However, Proposition 4.3 and the eigenvalue distribution of $A_{c l}$ are not sufficient to conclude about the stability of (8) for small values of $\vec{r}$ and $\vec{v}$, as an infinite number of characteristic roots may appear if the delays are increased from zero. The following two propositions provide information about the position of these introduced characteristic roots, and allow us to make assertions about preservation of (in)stability for small values of the delays.

Proposition 4.4 Assume that $\operatorname{det} \Delta_{D}(\lambda ; \vec{r}) \not \equiv 1$.

If $\Xi<0$, then there exist numbers $c>0$ and $\hat{\tau}>0$ such that for all $\vec{r} \in\left(\mathbb{R}^{+}\right)^{m} \backslash$ $\{\overrightarrow{0}\}$ and $\vec{v} \in\left(\mathbb{R}^{+}\right)^{p_{2}}$ with $\|\vec{r}\| \leq \hat{\tau}$ and $\|\vec{v}\| \leq \hat{\tau}$, the function $\operatorname{det} \Delta_{N}(\lambda ; \vec{r}, \vec{v})$ has exactly $n$ zeros in the half plane

$$
\left\{\lambda \in \mathbb{C}: \Re(\lambda)>-\frac{c}{\|\vec{r}\|}\right\} .
$$

If $\Xi>0$ then for all $\epsilon>0$, for all $\vec{r} \in\left(\mathbb{R}^{+}\right)^{m}$ with the components of $\vec{r}$ rationally independent, and for all $\vec{v} \in\left(\mathbb{R}^{+}\right)^{p_{2}}$, the function $\operatorname{det} \Delta_{N}(\lambda ; \vec{r}, \vec{v})$ has zeros in the half plane $\{\lambda \in \mathbb{C}: \Re(\lambda)>-\epsilon\}$. If, in addition, $\bar{C}_{D}\left(\vec{r}_{0}\right)>0$ 
for some $\vec{r}_{0} \in\left(\mathbb{R}_{0}^{+}\right)^{m}$, then for all $c>0, \hat{\tau}>0$ and $\nu \in\left(\mathbb{R}_{+}\right)^{n_{p}}$, there exist a vector $\vec{r} \in\left(\mathbb{R}_{0}^{+}\right)^{m}$ and a number $\lambda \in \mathbb{C}$ such that

$$
\|\vec{r}\| \leq \hat{\tau} \text {, det } \Delta_{N}(\lambda ; \vec{r}, \vec{v})=0 \text { and } \Re(\lambda)>c .
$$

Proposition 4.5 If $\operatorname{det} \Delta_{D}(\lambda ; \vec{r}) \equiv 1$, then there exist numbers $c>0$ and $\hat{\tau}>0$ such that for all $\vec{r} \in\left(\mathbb{R}^{+}\right)^{m} \backslash\{\overrightarrow{0}\}$ and $\vec{v} \in\left(\mathbb{R}^{+}\right)^{p_{2}}$ with $\|\vec{r}\| \leq \hat{\tau}$ and $\|\vec{v}\| \leq \hat{\tau}$, the function $\operatorname{det} \Delta_{N}(\lambda ; \vec{r}, \vec{v})$ has exactly $n$ zeros in the half plane

$$
\left\{\lambda \in \mathbb{C}: \Re(\lambda)>-\frac{c}{\|\vec{r}\|}\right\} .
$$

\section{Robust state derivative feedback}

Obviously, Equation (4) is of the general form (8) provided that

$$
\begin{aligned}
& p_{1}=n_{u} n, \quad p_{2}=n_{u} n, \quad m=n_{u}+n, \\
& \left(\tau_{1}, \ldots, \tau_{p_{1}}\right)=\left(\tau_{u_{1}}+\tau_{\dot{x}_{1}}, \ldots, \tau_{u_{1}}+\tau_{\dot{x}_{n}}, \ldots, \tau_{u_{n_{u}}}+\tau_{\dot{x}_{1}}, \tau_{u_{n_{u}}}+\tau_{\dot{x}_{n}}\right), \\
& \left(r_{1}, \ldots, r_{m}\right)=\left(\tau_{u_{1}}, \ldots, \tau_{u_{n_{u}}}, \tau_{\dot{x}_{1}}, \ldots, \tau_{\dot{x}_{n}}\right), \\
& \left(v_{1}, \ldots, v_{p_{2}}\right)=\left(\tau_{u_{1}}+\tau_{x_{1}}, \ldots, \tau_{u_{1}}+\tau_{x_{n}}, \ldots, \tau_{u_{n_{u}}}+\tau_{x_{1}}, \tau_{u_{n_{u}}}+\tau_{x_{n}}\right),
\end{aligned}
$$

and vectors $\vec{\gamma}_{k}, 1 \leq k \leq p_{1}$, and matrices $A_{0}, H_{k}, 1 \leq k \leq p_{1}$, are defined accordingly. In what follows, it is assumed that these substitutions have been made when using the terminology defined for general neutral equations in the previous section.

\subsection{Conditions on the gain parameters}

The following theorem presents conditions which guarantee robustness of (1)(2) under small feedback delays, the proof is provided in the appendix:

Theorem 5.1 Assume the system (1) is stabilized with the control law (2). If the feedback gain $K_{d}$ is such that

$$
\begin{aligned}
& \gamma_{0}\left(K_{d}\right):=\max \left\{\alpha\left(-\sum_{k=1}^{n_{u}} B E_{k} \sum_{l=1}^{n} K_{d} F_{l} e^{i\left(\mu_{k}+\nu_{l}\right)}\right):\right. \\
&\left.\vec{\mu} \in[0,2 \pi]^{n_{u}}, \vec{\nu} \in[0,2 \pi]^{n}\right\}<1,
\end{aligned}
$$

then the exponential stability of the closed-loop system is robust against small feedback delays. 
If $\gamma_{0}\left(K_{d}\right) \geq 1$, then the exponential stability of the closed-loop system is not robust against small delay perturbations. Furthermore, the closed-loop system is not exponentially stable whenever the delays $\vec{r}=\left(\tau_{u_{1}}, \ldots, \tau_{u_{n_{u}}}, \tau_{\dot{x}_{1}}, \ldots, \tau_{\dot{x}_{n}}\right)$ are rationally independent. If $\gamma_{0}\left(K_{d}\right)>1$, then for all $c>0$ and $\hat{\tau}>0$, there exist a vector $\vec{r} \in\left(\mathbb{R}_{0}^{+}\right)^{m}$ and a number $\lambda \in \mathbb{C}$ such that $\|\vec{r}\| \leq \hat{\tau}$, $\operatorname{det} \Delta_{N}(\lambda ; \vec{r}, \overrightarrow{0})=$ 0 and $\Re(\lambda)>c$.

\subsection{Filtered proportional-derivative feedback control}

The condition (22) imposes a constraint on the choice of the gain $K_{d}$ and may make robust stabilization impossible. If this condition is not satisfied then small delays may introduce unstable characteristic roots, which have a large modulus for small values of the delays (following from Rouché type arguments, similar to the one used in the proof of Proposition 4.3). An intuitive solution to prevent large unstable roots could be to include a low pass filter in the derivative control loop. This will be investigated in what follows. Note that such an approach has already been successfully applied to the discretization of distributed delay controllers in the context of finite spectrum assignment where similar robustness problems occur (Michiels, et al., 2004; Mondié and Michiels, 2003).

When applying a first order filter to (2), the controller becomes:

$$
T \dot{u}(t)+u(t)=-K_{p} x(t)-K_{d} \dot{x}(t)
$$

where $T=1 / \omega_{f}$ is the time constant of the filter, and $\omega_{f}$ is its cutoff frequency. The feedback system that consists of (1) and (23) is given by

$$
\dot{z}(t)=\left[\begin{array}{cc}
A & B \\
-\frac{1}{T} K_{p} & -\frac{1}{T} I
\end{array}\right] z(t)+\left[\begin{array}{cc}
0 & 0 \\
-\frac{1}{T} K_{d} & 0
\end{array}\right] \dot{z}(t),
$$

where $z(t)=\left[x(t)^{T} u(t)^{T}\right]^{T}$.

Consider again that delays $\left(\tau_{u_{1}}, \ldots, \tau_{u_{n_{u}}}\right)$ have appeared in the inputs of the system, delays $\left(\tau_{x_{1}}, \ldots, \tau_{x_{n}}\right)$ in the measurements of the components of $x(t)$ and delays $\left(\tau_{\dot{x}_{1}}, \ldots, \tau_{\dot{x}_{n}}\right)$ in the measurements of the components of $\dot{x}(t)$. Then, 
the feedback system changes from (24) to

$$
\begin{array}{r}
\dot{z}(t)+\sum_{k=1}^{n}\left[\begin{array}{cr}
0 & 0 \\
\frac{1}{T} K_{d} F_{k} & 0
\end{array}\right] \dot{z}\left(t-\tau_{\dot{x}_{k}}\right)= \\
{\left[\begin{array}{cc}
A & 0 \\
0 & -\frac{1}{T} I
\end{array}\right] z(t)+\sum_{k=1}^{n_{u}}\left[\begin{array}{cc}
0 & B E_{k} \\
0 & 0
\end{array}\right] z\left(t-\tau_{u_{k}}\right)+} \\
\sum_{k=1}^{n}\left[\begin{array}{cc}
0 & 0 \\
-\frac{1}{T} K_{p} F_{k} & 0
\end{array}\right] z\left(t-\tau_{x_{k}}\right), \quad(25)
\end{array}
$$

with $E_{k}, 1 \leq k \leq n_{u}$ and $F_{k}, 1 \leq k \leq n$ defined in (5). This system corresponds to the degenerate case where $\operatorname{det} \Delta_{D}(\lambda ; \vec{r}, \vec{v}) \equiv 1$. If $K_{d}$ and $T$ are such that the delay free system is asymptotically stable, then by Proposition 4.3 and Proposition 4.5, stability is preserved for small values of the delays.

Remark 5 Although stability is always preserved for sufficiently small delays, the maximal allowable delays tend to zero as $T \rightarrow \infty$ whenever $\gamma_{0}\left(K_{d}\right)>1$, following from Rouché type arguments as spelled out in the proof of Proposition 4.3. This implies an inherent trade-off in determining the cut-off frequency of the filter: in order not to affect the nominal, delay free behavior too much the cut-off frequency should be sufficiently large. However, if the cut-off frequency is too large, then the delay margin may be unacceptably small. This trade-off will become apparent in the next section.

\section{Case studies}

Two case study examples are presented with the focus on the stability issues related with the application of the state derivative feedback.

\subsection{Vibration suppression of a platform}

As the first application example, the vibration suppression of a platform is adopted, which has been presented in Abdelaziz and Valášek (2005a) and where the state derivative feedback, i.e. the control law (2) with $K_{p}=0$, was used to control the system shown in Fig. 3. A linear state space formulation of the system, with states $x=\left[\begin{array}{llll}x_{1} & x_{2} & \dot{x}_{1} & \dot{x}_{2}\end{array}\right]$ (assuming $\varphi$ to be small), is given by (1) with the matrices 


$$
A=\left(\begin{array}{cccc}
0 & 0 & 1 & 0 \\
0 & 0 & 0 & 1 \\
-k_{1} c_{1}-k_{2} c_{2}-b_{1} c_{1} & b_{2} c_{2} \\
-k_{1} c_{2}-k_{2} c_{1} & -b_{1} c_{2} & b_{2} c_{1}
\end{array}\right), B=\left(\begin{array}{cc}
0 & 0 \\
0 & 0 \\
c_{1} & c_{2} \\
c_{2} & c_{1}
\end{array}\right),
$$

where $c_{1}=\frac{1}{m}+\frac{L^{2}}{I}, c_{2}=\frac{1}{m}-\frac{L^{2}}{I}, y=\frac{\left(x_{1}+x_{2}\right)}{2}$ and $\varphi=\frac{x_{1}-x_{2}}{2 L}$. The parameters $m$ and $I$ represent the mass and moment of inertia, the parameters $k_{1}$ and $k_{2}$ are the spring constants, $b_{1}$ and $b_{2}$ are the damping constants, $2 L$ is the distance between the two supports, $\varphi$ is the angle of inclination of the mass with the horizontal, $y$ is the displacement of the center of the mass, $x_{1}$ and $x_{2}$ are the displacements of the sides of the mass and $u_{1}$ and $u_{2}$ are the control inputs.

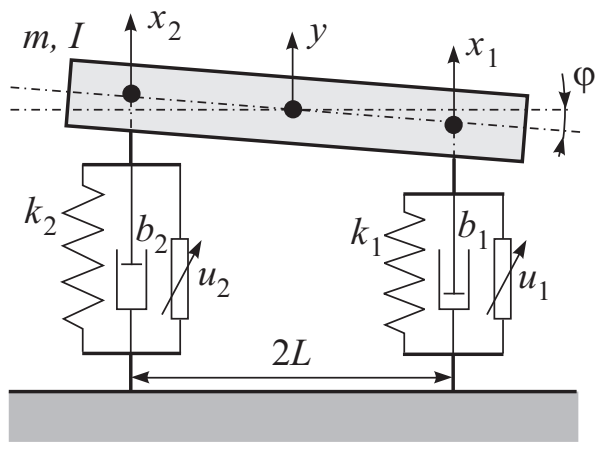

Fig. 3. Scheme of the vibration suppression control of a platform

The model parameters are given as $m=10 \mathrm{~kg}, I=1 \mathrm{kgm}^{2}, L=1 \mathrm{~m}, k_{1}=$ $500 \mathrm{~N} / \mathrm{m}, k_{2}=700 \mathrm{~N} / \mathrm{m}, b_{1}=10 \mathrm{Ns} / \mathrm{m}$ and $b_{2}=20 \mathrm{Ns} / \mathrm{m}$. It should be noted that the open loop system roots are $\lambda_{1,2}=-15.14 \pm 31.17 i, \lambda_{3,4}=$ $-1.36 \pm 10.71 i$, which implies considerably underdamped system responses.

In Abdelaziz and Valášek (2005a) a derivative feedback controller has been designed using an LQR method, resulting in the gain

$$
K_{d}=\left(\begin{array}{cccc}
98.008 & -6.527 & 1.588 & -1.512 \\
-4.662 & 35.641 & -0.298 & 1.949
\end{array}\right) .
$$

Remark 6.1 Since the velocities that correspond to the positions $x_{1}$ and $x_{2}$ are state variables of the system, the control action of pure derivative feedback can also be considered as the action of the PD state feedback (2) with the matrices $K_{p}=\left[0, K_{d 1}\right]$ and $K_{d}=\left[0, K_{d 2}\right]$, where $K_{d 1}$ and $K_{d 2}$ are square submatrices of the gain $K_{d}=\left[K_{d 1}, K_{d 2}\right]$ given here by (27).

With the control law (2) with $K_{p}=0$ and $K_{d}$ given by (27) the char- 
acteristic roots of the closed loop system are $\lambda_{1}=-23.19, \lambda_{2}=-5.94$ and, $\lambda_{3,4}=-5.63 \pm 10.12 i$. The transient response from an initial state $x(0)=[-0.01,0.02,-0.02,0.01]^{T}$ can be seen in Fig. 4 . The system response shows desirable characteristics, namely a settling time of approximately $0.5 \mathrm{~s}$.
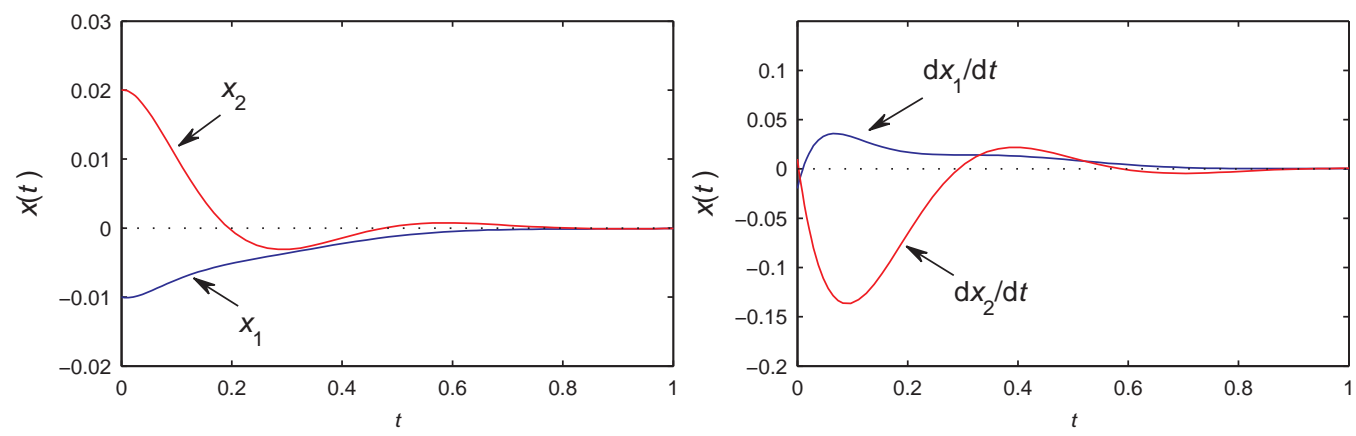

Fig. 4. Response of the state feedback system (1)-(2) with the matrices (26) and the gains $K_{p}=0$ and $K_{d}$ given by (27).
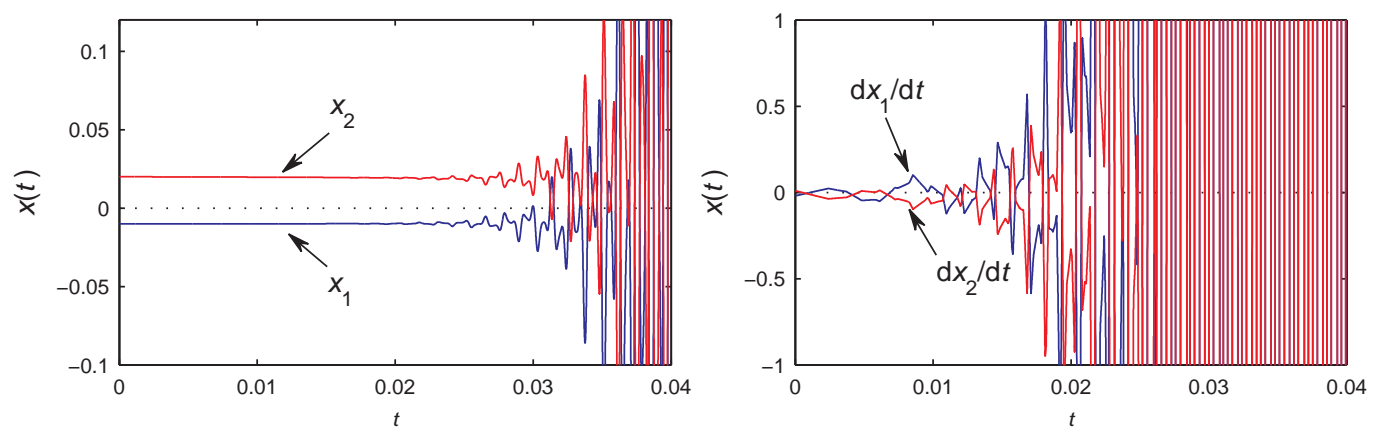

Fig. 5. Response of the feedback system (4) with the matrices (26), delays (29) and gains $K_{p}=0$ and $K_{d}$ given by (27).
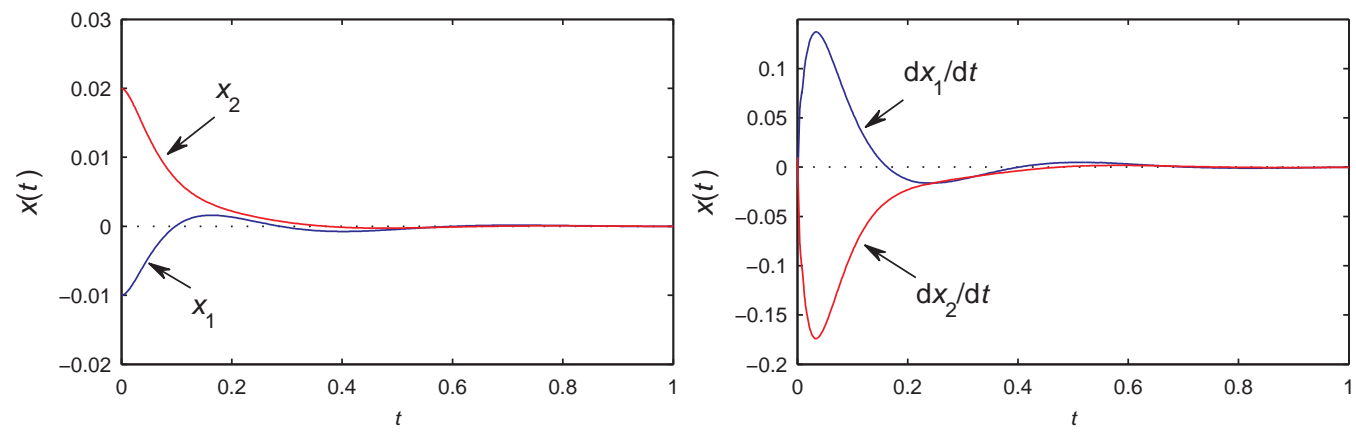

Fig. 6. Response of the state derivative feedback system (4) with the matrices (26) feedback gains $K_{p}=0$ and $K_{d}$ given by (30), and delays (29).

First, the effect of small delay perturbations at the system's inputs and states are studied for the derivative feedback system with the gain (27). If the delays appear, then the closed loop system becomes a neutral system of the form (4). 
According to (21), the closed loop system has $p_{1}=8$ delays in the difference part that depend on $m=6$ delays. However, due to the special form of the matrix

$$
B K_{d}=\left(\begin{array}{cc}
0 & 0 \\
B_{2} K_{d 1} & B_{2} K_{d 2}
\end{array}\right),
$$

where $B_{2}$ is a square submatrix of $B:=\left[0, B_{2}^{T}\right]^{T}$ and $K_{d 1}, K_{d 2}$ are defined as in Remark 6.1, the matrix $K_{d 1}$ has no influence on the robustness indicator $\gamma_{0}$, defined in Theorem 5.1. This implies that the stability is robust against small delays in the measurements of the velocities, $\tau_{\dot{x}_{1}}, \tau_{\dot{x}_{2}}$, which is expected from Remark 6.1. An application of Theorem 5.1 reveals that the closed-loop system is not robustly stable against small delays, because

$$
\gamma_{0}=5.3 .
$$
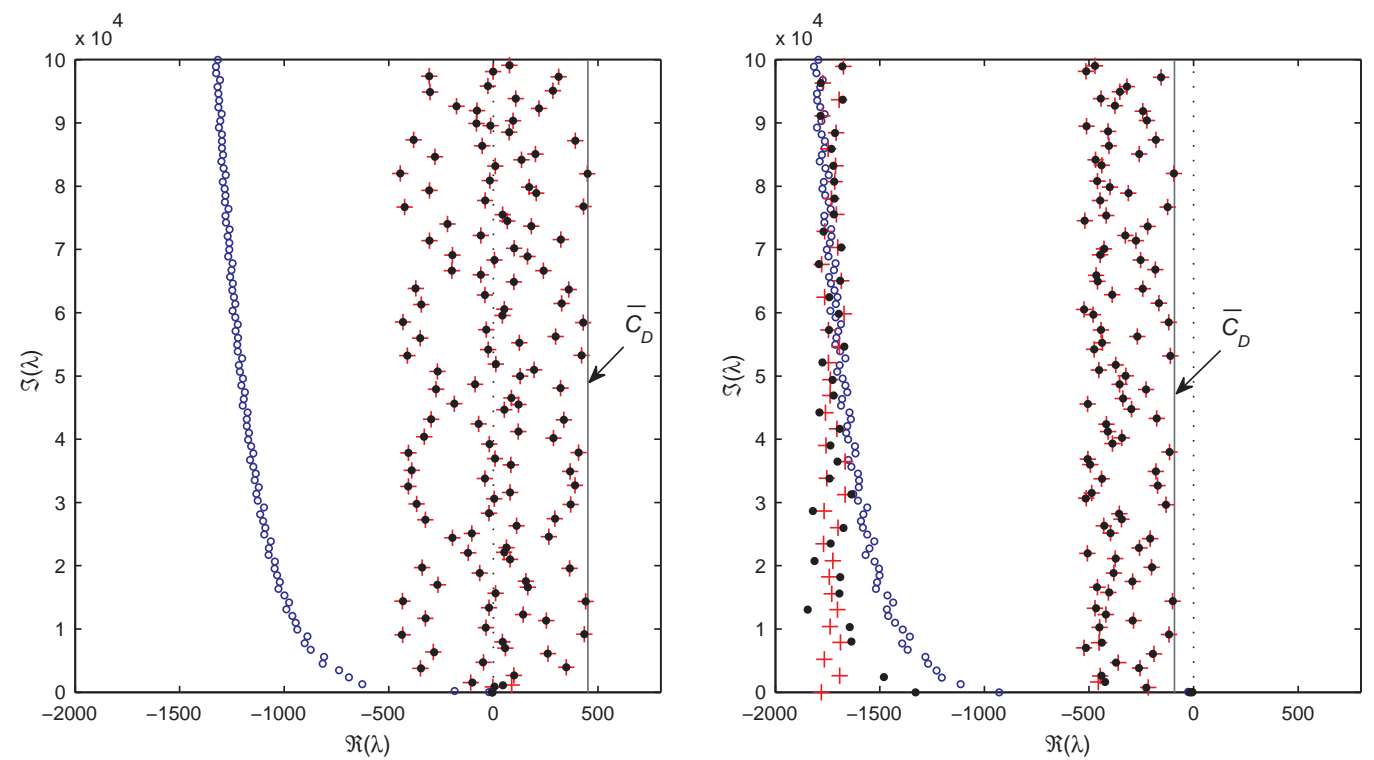

Fig. 7. Characteristic roots of the feedback system (1)-(2) with the matrices (26) and the feedback gains $K_{p}=0$ and $K_{d}$, left given by (27) and right given by (30). Dots - characteristic roots of the feedback system with small delays (29) given by $(4),+-$ characteristic roots of the associated difference equation to the system (4) with delays (29), circles - characteristic roots of the feedback system with a filter in the feedback, given by (25), with $T=0.05$ and with delays (29).

Even though the feedback system with the gain (27) may happen to be stable for certain combinations of fixed rationally dependent delays, such stability has no practical meaning. It is due to the fact that even infinitesimal changes of such delays may destroy the stability. Moreover, the stability is lost whenever the delays happen to be rationally independent. To demonstrate this, consider the following small rationally independent delays 

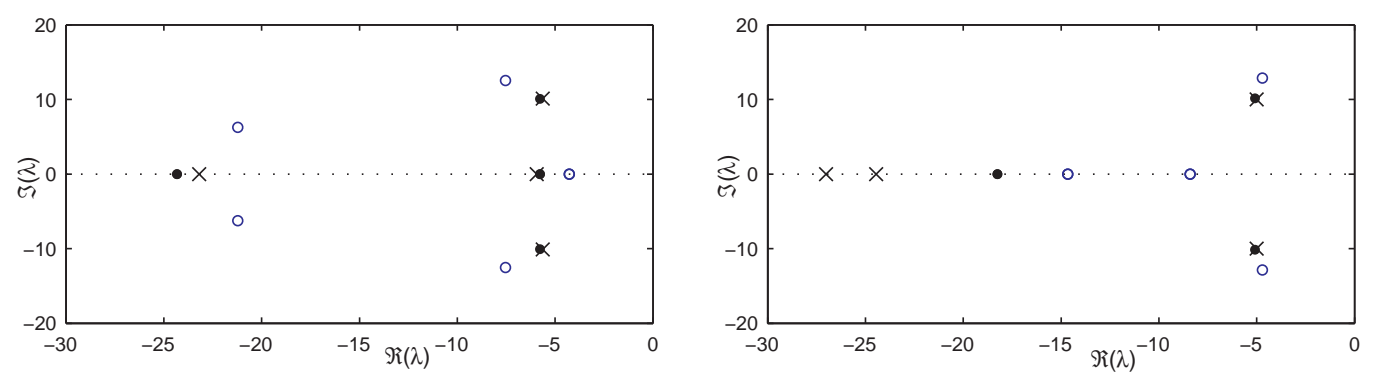

Fig. 8. Detail of Fig. 7 - characteristic roots of the feedback system (1)-(2) with the matrices (26) and the feedback gains $K_{p}=0$ and $K_{d}$, left given by $(27)$ and right given by (30). $\times$ - characteristic roots of the delay free feedback system, dots - characteristic roots of the feedback system with small delays (29) given by (4), circles - characteristic roots of the feedback system with a filter in the feedback, given by (25), with $T=0.05$ and with delays (29).
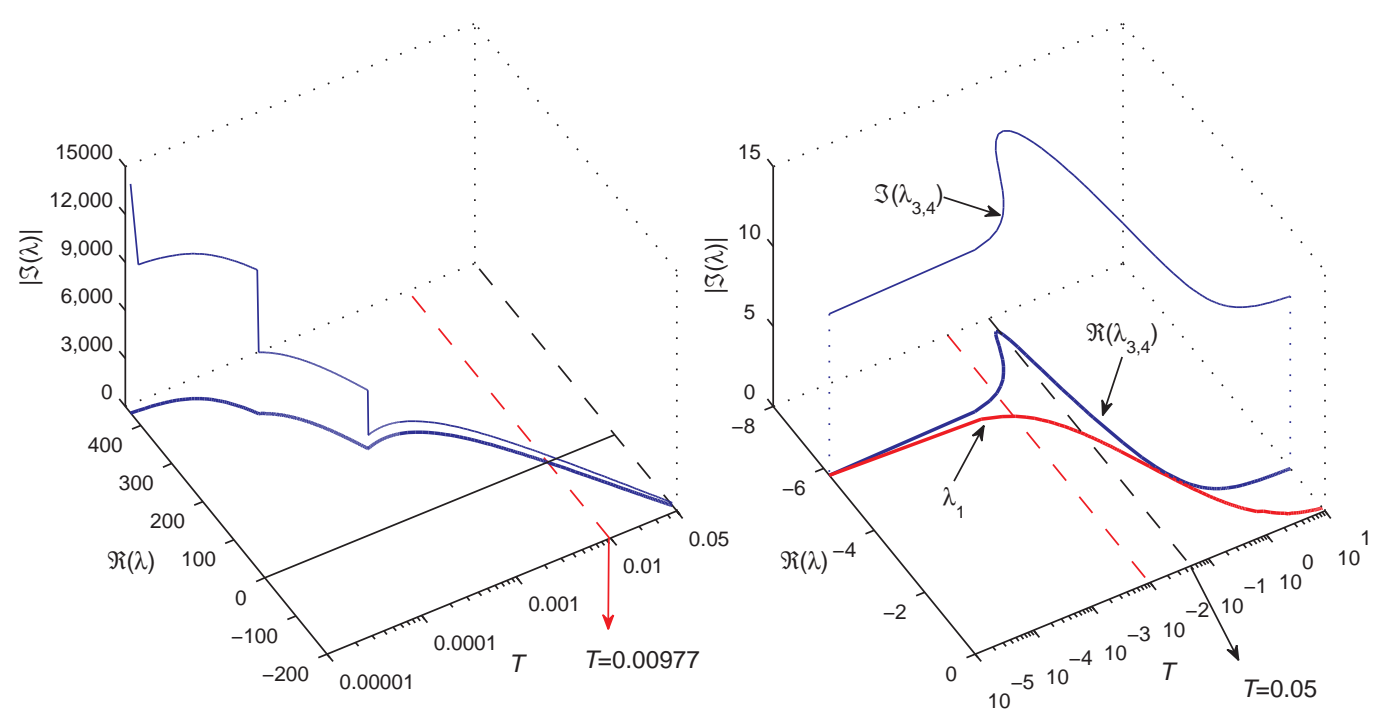

Fig. 9. Position of the rightmost characteristic roots in the high and low frequency ranges of the spectrum of the feedback system with a filter in the feedback, given by (25), with the matrices (26), gains $K_{p}=0$ and $K_{d}$ given by (27), delays (29) with respect to the value of $T$. Left - real (thick line) and imaginary (thin line) parts of the rightmost roots for $\Im(\lambda)>20$. Right - rightmost roots in the low frequency range, i.e. for $\Im(\lambda) \leq 20$.

$$
\begin{gathered}
\tau_{u_{1}}=10^{-3}, \tau_{\dot{x}_{1}}=\sqrt{5} 10^{-3}, \tau_{\dot{x}_{3}}=\sqrt{2} 10^{-3} \\
\tau_{u_{2}}=\pi 10^{-3}, \tau_{\dot{x}_{2}}=\sqrt{3} 10^{-3}, \tau_{\dot{x}_{4}}=e 10^{-3}
\end{gathered}
$$

The characteristic roots of the derivative feedback system with the feedback gain (27) and the above delays are shown in Fig. 7 and Fig. 8, respectively, in the left parts of the figures. Let us remark that the roots have been computed by the rootfinding algorithm designed in Vyhlídal and Zítek (2003). As can be 
seen, even though the prescribed roots shown in the detailed Fig. 8 did only move slightly due to delay perturbations, a large number of unstable roots appeared (in fact infinitely many), bounded from the right by the safe upper bound $\bar{C}_{D}=451.6,\left(\bar{C}_{D}\right.$ has been computed according to Theorem 4.1). In Fig. 5 the response of the feedback system is shown for the given delays (29). As can be seen, the stability is lost due to high frequency oscillations. The high frequencies imply that the oscillations of $\dot{x}$ have a much larger amplitude than these of $x\left(x(t) \sim e^{(\sigma+j \Omega) t}, \Omega>>0 \Rightarrow \dot{x}(t) \sim(\sigma+j \Omega) x(t)\right)$. This explains the seemingly 'delayed onset of instability' of $x(t)$ compared to $\dot{x}(t)$ in the figure. The frequencies of the unstable oscillations are determined by both the size and the interdependence of the delays as they originate from the difference part of the system equations. Note that the characteristic roots introduced by the delay presence have to move off to infinity as all delays tend to zero, thus small delay values imply that characteristic roots can only cross the imaginary axis at high frequencies.

As the second task, the filtered derivative feedback (23) is applied to control the system (with $K_{d}$ given by $(27)$ and $K_{p}=0$ ). If the delays appear at the inputs and measurements, the feedback system becomes a degenerate neutral system of the form (25). According to Proposition 4.3 and Proposition 4.5, the stability of such system is preserved for small delay perturbations. In Fig. 9, the positions of the rightmost roots in the high frequency range, i.e. $\Im(\lambda)>20$, and in the low frequency range are shown for the feedback system with the gain (27) and with delays (29) w.r.t. the value of $T$. As can be seen in the left part of the figure, increasing the value of $T$ results in shifting the high frequency rightmost roots to the left. It also results in decreasing the imaginary parts of the rightmost roots. Consequently, the feedback system is stabilized for $T=0.00977,\left(\omega_{f}=102.35\right)$. For $T=0.05,\left(\omega_{f}=20\right)$, the high frequency rightmost roots acquire the position $\lambda_{6,7}=-185.4 \pm 173.7 i$ while the dominant roots are $\lambda_{1}=-4.27 \lambda_{2,3}=-7.54 \pm 12.53 i$ and $\lambda_{4,5}=-21.22 \pm 6.24 i$, see Fig. 7. For larger values of $T$, the roots $\lambda_{1}$ and $\lambda_{2,3}$ move further to the right, see Fig. 9, which results in slower and less damped system's responses than those for $T=0.05$.

As the last task, a controller of the form (7) was sought which satisfies the robustness condition (22) of Theorem 5.1. Since this condition is affected by the submatrix $K_{d 2}$ only (see (28)), finding the four coefficients in

$$
K_{d}=\left(K_{d 1} K_{d 2}\right)
$$

that provide us robustness against small delays is an easy optimization task. The remaining four parameters of the submatrix $K_{d 1}$ in (30) are obtained from prescribing the characteristic roots $\lambda_{1}=-24.45, \lambda_{2}=-27.01$ and, 
$\lambda_{3,4}=-5 \pm 10 i$ to the closed loop system. This results in the gain matrix

$$
K_{d}=\left(\begin{array}{cccc}
19.64 & 5.899 & 0.220 & -0.210 \\
8.367 & 49.0 & -0.150 & 0.120
\end{array}\right),
$$

for which $\gamma_{0}=0.7$ in Theorem 5.1. As can be seen in Fig. 6 such a gain setting provides fast and well damped response. The characteristic roots of the feedback system for the gain (30) and the rationally independent delays (29) are shown in Fig. 7 and Fig. 8. As can be seen, the characteristic roots of the associated difference equation are bounded from the right by the safe upper bound $\bar{C}_{D}=-91.4$. To the right of $\bar{C}_{D}$, only four stable roots are located that are shown in the Fig. 8. Even though the feedback system is robustly stable with respect to small delays without using the filter, the filtered feedback (23) with $T=0.05$ and $K_{p}=0$ has also been tested and the characteristic roots of the filtered feedback system are also shown in the right parts of Fig. 7 and Fig. 8, respectively. As can be seen, the infinite chain of roots is located much further to the left than $\bar{C}_{D}$. Due to this and also due to retarded like distribution of the roots, the risk of stability loss due to large frequency root crossings of the stability boundary is significantly reduced compared to unfiltered feedback.

\subsection{Robustness analysis of regenerative chatter control in turning process}

In the second case study example, it is demonstrated that the robustness analysis with respect to small delays can also be applied to a system that is nominally a time delay system. Thus, next to small delays originated in the feedback loops, the system has delays inherent to its nominal dynamics.

The practical problem under consideration is a suppression of a regenerative chatter in turning process. This regenerative chatter, which has been analysed e.g. in Stepan (2001) and recently in Insperger, Barton, and Stepan (2008) is undesirable due to its adverse effects on surface finish, machining accuracy and also tool life. Because of some external perturbations, the tool starts a damped oscillation relative to the workpiece. After a revolution of the workpiece, the chip thickness will vary at the tool due to this wavy surface. Therefore the cutting force is dependent on the actual and delayed values of the displacement of the tool. The delay $\tau$ is equal to the period of revolution of the workpiece $\tau=\frac{2 \pi}{\Omega}$, where $\Omega$ is the angular velocity of the workpiece.

Fig. 10 shows a schematic of the regenerative chatter model in question. The tool is assumed to be compliant in axis $y$ while it is assumed to be rigid in axis $x$. Also the workpiece is assumed to be rigid. It is known that cutting force is given by $F_{y}(w, h)=K_{y} w h^{3 / 4}$, where $K_{y}$ is the cutting coefficient, $w$ 


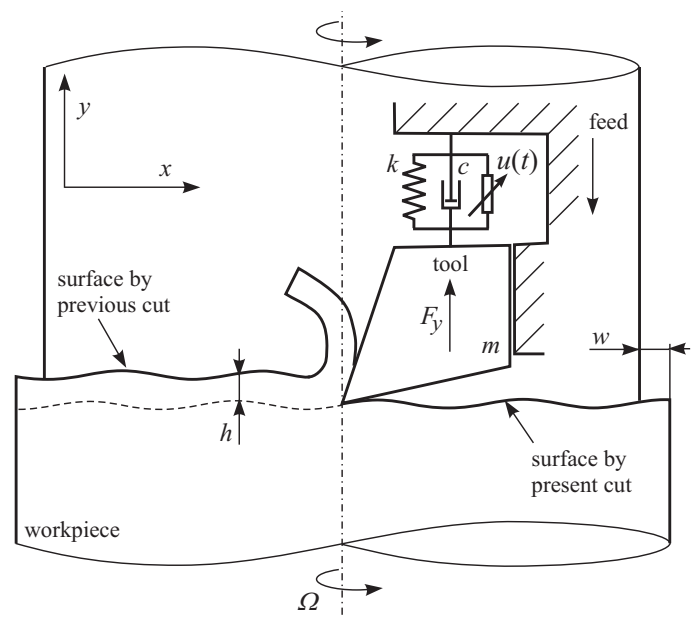

Fig. 10. A schematic of regeneration in turning process

is the depth of cut and $h=h_{0}+y(t-\tau)-y(t)$ is the chip thickness with $h_{0}$ to be its steady value. Introducing parameter $\kappa=\left.\frac{\partial F_{y}(w, h)}{\partial h}\right|_{h_{0}}=\frac{3}{4} K_{y} w h_{0}^{-1}$, the displacements of the tool in the $y$ axis can be modeled as a 1 DOF oscillator (Stepan, 2001; Insperger, Barton, and Stepan, 2008)

$$
m \ddot{y}(t)+c \dot{y}(t)+k y(t)=\kappa(y(t-\tau)-y(t))+u(t)
$$

where $m, c, k$ are the modal mass, the damping and stiffness parameters and $u$ is the external force - the control variable of the system (exerted e.g. by a piesoactuator).

In order to damp the regenerative oscillations of the tool, the following purely dynamical controller is considered

$$
u(t)=-k_{1} \dot{y}(t)-k_{2} \ddot{y}(t),
$$

as it is supposed that an accelerometer and a velocitymeter are used for measuring motion of the tool.

Considering the closed loop system (31)-(32) and the small delays in the feedback loop as in (4), i.e. $\tau_{u}$ at the input, and $\tau_{\dot{y}}, \tau_{\ddot{y}}$ at measurements, the governing equations can be expressed in the state space form as

$$
\dot{x}+H_{1} \dot{x}\left(t-\tau_{u}-\tau_{\ddot{y}}\right)=A_{0} x(t)+A_{1} x(t-\tau)+A_{2} x\left(t-\tau_{u}-\tau_{\dot{y}}\right)
$$


where $x=\left[\begin{array}{ll}y & \dot{y}\end{array}\right]^{T}$ and

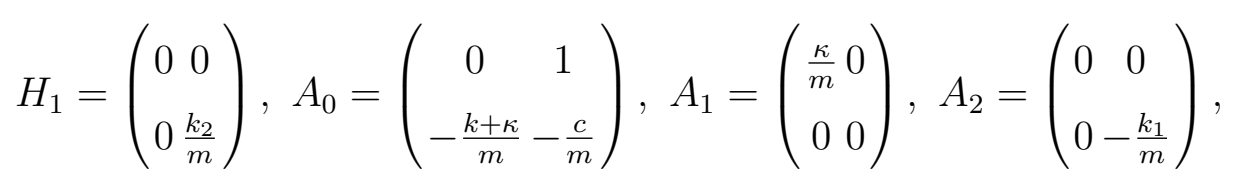

which is a neutral system of the form (8). According to the Theorem 5.1, the system is robust against small feedback delays if $\gamma_{0}<1$. Due to the fact there is only one delay combination in the neutral part, $\gamma_{0}$ reduces to

$$
\gamma_{0}:=\max \left\{\alpha\left(-H_{1} e^{i \theta}\right): \theta \in[0,2 \pi]\right\}=\rho\left(H_{1}\right)=\frac{\left|k_{2}\right|}{m} .
$$

Thus, the above inequality is satisfied for $\left|k_{2}\right|<m$, which is the condition under which the system's stability is insensitive to small feedback delays.

If $\left|k_{2}\right|>m$ then the sensitivity problem with respect to delays can be solved by including a filter in the control loop:

$$
T \dot{u}(t)+u(t)=-k_{1} \dot{y}-k_{2} \ddot{y},
$$

as proven in subsection 5.2. However a filter modifies the delay free system, and as in the previous example, tuning the parameter $T$ may involve a trade-off between performance and delay robustness.

\section{Conclusions}

It has been pointed out that the use of control laws involving state derivative feedback may lead to an extreme sensitivity of closed-loop stability w.r.t. small delays that exist at least by virtue of inescapable latencies in sensor or actuator responses. The stability problem originates from the fact that state derivative feedback does not lead to a retarded type but to a neutral type system if unmodelled feedback delays are taken into account. Hence, the user has to be cautious in including the derivative action in the state feedback and has to wonder not only whether the matrix $\left[I+B K_{d}\right]$ in $(3)$ is regular but primarily whether the condition for robustness against small delay perturbations given by Theorem 5.1 is satisfied. Thus, although Proposition 3.2 suggests a direct similarity between proportional and pure state derivative feedback, the use of state derivative action substantially modifies the nature of the system, which becomes apparent in the robust stability analysis. If a state feedback control system including derivative action does not comply with the robust stability condition (22), then the asymptotic stability of the closed loop system is not robust against arbitrarily small delays, and the application of the 
control law turns out to be infeasible. It has also been shown that including a low-pass filter in the control loop represents a natural remedy. Even though the filtered feedback system is still a neutral system, due to its degenerate form the possible fragility of stability, in the sense of non-robustness against small delays, is removed.

The vibration control application has demonstrated the possible extreme sensitivity of state derivative feedback w.r.t. apparently negligible delays, as well as how this problem can be overcome by including a filter or taking into account a robustness constraint on the gain parameters in the design. Let it be noticed that, owing to the fact that a derivative action cannot fix any reference state, pure derivative state feedback can be applied only in cases where a reference output value is not required. For example, it can be used in applications where an inner-control loop is used for damping the vibration, whereas the outer control loop performs the control. Such a control scheme has recently been used, e.g., in control of the hard disk drives in Horowitz et al. (2007).

As a by-product the stability theory for neutral equations with arbitrarily many mutually dependent small delays has been developed, with the emphasis on the asymptotic behavior of the characteristic roots when the delays are brought to zero.

\section{Acknowledgments}

This research has been supported by the Ministry of Education of the Czech Republic under the Project 1M0567, by the Belgian Programme on Interuniversity Poles of Attraction, initiated by the Belgian State, Prime Ministers Office for Science, Technology and Culture, and by OPTEC, the Center of Excellence on Optimization in Engineering of the K.U.Leuven. Wim Michiels is a postdoctoral fellow of the Fund for Scientific Research -Flanders (Belgium).

\section{Appendix}

Proof of Proposition 4.3

The function $\operatorname{det} \Delta_{N}(\lambda ; \vec{r}, \vec{v})$ uniformly converges on compact subsets of $\mathbb{C}$ to

$$
\operatorname{det} \tilde{\Delta}(\lambda):=\operatorname{det}\left(\lambda\left(I+\sum_{k=1}^{p_{1}} H_{k}\right)-A_{0}-\sum_{k=1}^{p_{2}} A_{k}\right)
$$


as $(\vec{r}, \vec{v}) \rightarrow(\overrightarrow{0}, \overrightarrow{0})$. Let $\mathcal{B} \subset \mathbb{C}$ be an open disk which contains the zeros $\left(\mu_{1}, \ldots, \mu_{n}\right)$ of $\operatorname{det} \tilde{\Delta}$. The above convergence property implies the existence of a number $\hat{\tau}$ such that

$$
\begin{aligned}
& \max _{\lambda \in \partial \mathcal{B}}\left|\operatorname{det} \tilde{\Delta}(\lambda)-\operatorname{det} \Delta_{N}(\lambda ; \vec{r}, \vec{v})\right|<\min _{\lambda \in \partial \mathcal{B}}|\operatorname{det} \tilde{\Delta}(\lambda)|, \\
& \forall \vec{r} \in[0, \tilde{\tau}]^{m}, \forall \vec{v} \in[0, \hat{\tau}]^{n_{p}} .
\end{aligned}
$$

By Rouché's Theorem one concludes that $\operatorname{det} \Delta_{N}(\lambda ; \vec{r}, \vec{v})$ and $\operatorname{det} \tilde{\Delta}(\lambda)$ have the same number of zeros in $\mathcal{B}(n)$ if $\vec{r} \in[0, \hat{\tau}]^{m}$ and $\vec{v} \in[0, \hat{\tau}]^{p_{2}}$. The existence of the functions $\tilde{\lambda}_{i}, 1 \leq i \leq n$, follows from the combination of this result with the continuity of the individual zeros of $\operatorname{det} \Delta_{N}$ w.r.t. the delay parameters $\vec{r}$ and $\vec{v}$ (see Hale and Verduyn Lunel (1993)). The assertion (20) can be shown in a similar way, by letting $\mathcal{B}$ be a disk with (arbitrarily small radius) centered at a zero of $\operatorname{det} \tilde{\Delta}$, and taking the same steps.

\section{Proof of Proposition 4.4}

First, consider the case where $\Xi<0$. The proof is based on a scaling of the complex plane. Let

$$
p(\mu ; \tilde{\tau}, \vec{r}, \vec{v}):=\tilde{\tau}^{n} \operatorname{det} \Delta_{N}\left(\frac{\mu}{\tilde{\tau}} ; \vec{r}, \vec{v}\right)
$$

where $\tilde{\tau} \in(0,1)$. Consider

$$
p(\mu ; \tilde{\tau}, \vec{r}, \vec{v})=\operatorname{det}\left(\mu\left(\Delta_{D}\left(\mu ; \frac{\vec{r}}{\tilde{\tau}}\right)\right)-\tilde{\tau}\left(A_{0}+\sum_{k=1}^{p_{2}} A_{k} e^{-\mu \frac{v_{k}}{\tilde{\tau}}}\right)\right) .
$$

Let $c>0$ be such that

$$
\sup \left\{\bar{C}_{D}(\vec{\rho}):\|\vec{\rho}\| \leq 1\right\}<-c
$$

Note that such a number $c$ always exists as Proposition 2 holds and a sequence $\left\{\vec{\rho}^{(j)}\right\}_{j \geq 1}$ satisfying $\vec{\rho}^{(j)} \in\left(\mathbb{R}_{0}^{+}\right)^{m}$ and

$$
\lim _{j \rightarrow \infty} \bar{C}_{D}\left(\bar{\rho}^{(j)}\right)=0
$$

cannot occur because it would imply that $\bar{C}_{D}(\vec{r}) \geq 0$ for all $\vec{r} \in\left(\mathbb{R}^{+}\right)^{m}$. If $p\left(\mu_{0} ; \tilde{\tau}, \vec{r}, \vec{v}\right)=0$ with $\|\vec{r}\|<\tilde{\tau}$ and $\|\vec{v}\|<\tilde{\tau}$, and if $\Re\left(\mu_{0}\right) \geq-c$, then

$$
\left|\mu_{0}\right| \leq \tilde{\tau} d
$$


where

$$
d:=\max _{\|\vec{\rho}\| \leq 1, \Re(\mu) \geq-c}\left\|\left(\Delta_{D}(\mu ; \vec{\rho})\right)^{-1}\right\|\left(\left\|A_{0}\right\|+\max _{\|\vec{\sigma}\| \leq 1} \sum_{k=1}^{p_{2}}\left\|H_{k}\right\| e^{c \sigma_{k}}\right) .
$$

Hence, if $\|\vec{r}\|<\tilde{\tau}$ and $\|\vec{v}\|<\tilde{\tau}$, then all zeros of $p$ in the half plane

$$
\{\mu \in \mathbb{C}: \Re(\mu) \geq-c\}
$$

lie in the compact set

$$
\mathcal{P}:=\{\mu \in \mathbb{C}: \Re(\mu) \geq-c \text { and }|\mu| \leq d\}
$$

(where $\tilde{\tau}<1$ ). Next, determine $\hat{\tau}>0$ such that

$$
\begin{aligned}
\min _{\mu \in \partial \mathcal{P}}\left|\mu^{n}-\operatorname{det}\left(\mu I-\tau\left(\Delta_{D}(\mu ; \vec{\rho})\right)^{-1}\left(A_{0}+\sum_{k=1}^{p_{2}} A_{k} e^{-\mu \sigma_{k}}\right)\right)\right| & \\
& <\max _{\mu \in \partial \mathcal{P}}\left|\mu^{n}\right|
\end{aligned}
$$

for all $\tau \in(0, \hat{\tau}]$, for all $\vec{\rho} \in \mathbb{R}^{m}$ and $\vec{\sigma} \in \mathbb{R}^{p_{2}}$ with $\|\vec{\rho}\| \leq 1$ and $\|\vec{\sigma}\| \leq 1$. By Rouché's Theorem it follows that under the condition $\tilde{\tau}<\hat{\tau}, p(\cdot ; \tilde{\tau}, \vec{r}, \vec{v})$ has the same number of zeros in $\mathcal{P}$ as the function $\mu^{n}$, thus $n$, for all $\vec{r} \in\left(\mathbb{R}_{0}^{+}\right)^{m}$ and $\vec{v} \in\left(\mathbb{R}^{+}\right)^{p_{2}}$ with $\|\vec{r}\| \leq \tilde{\tau}$ and $\|\vec{v}\| \leq \tilde{\tau}$. Consequently, $p$ has exactly $n$ zeros in the right half plane $\{\mu \in \mathbb{C}: \Re(\mu) \geq-c\}$, thus, det $\Delta_{N}(; \vec{r}, \vec{v})$ has $n$ zeros in the half plane $\{\lambda \in \mathbb{C}: \Re(\lambda) \geq c / \tilde{\tau}\}$, which implies the statement of the proposition.

Next, consider the case where $\Xi>0$. The first assertion follows from $c_{D}(\vec{r})=$ $\bar{C}_{D}(\vec{r})$ for rationally independent $\vec{r}$ (Proposition 2) and $\bar{C}_{D}(\vec{r}) \geq 0$. Concerning the latter, by definition $\bar{C}_{D}\left(\vec{r}_{0}\right)>0$ implies the existence of a vector $\vec{s}_{0} \in$ $\left(\mathbb{R}_{0}^{+}\right)^{m}$ for which $c_{D}\left(\vec{s}_{0}\right)>0$. Because

$$
c_{D}\left(\frac{\vec{s}_{0}}{\tilde{\tau}}\right)=\frac{c_{D}\left(\vec{s}_{0}\right)}{\tilde{\tau}}
$$

for all $\tilde{\tau}>0$ and because of (13), the function $\Delta_{N}\left(\cdot ; \frac{\vec{s}_{0}}{\tilde{\tau}}, \vec{\nu}\right)$ has for all $\nu \in$ $\left(\mathbb{R}^{+}\right)^{p_{2}}$ zeros with real part larger or equal to $\frac{c_{D}\left(\vec{s}_{0}\right)}{\tilde{\tau}}$. The assertion of the proposition directly follows.

\section{Proof of Proposition 4.5}

As $\operatorname{det} \Delta_{D}(\lambda ; \vec{r}) \equiv 1$, the zeros of $\operatorname{det} \Delta_{N}(\lambda ; \vec{r}, \vec{v})$ are equal to the zeros of

$$
Q(\lambda ; \vec{r}, \vec{v}):=\operatorname{det}\left(\lambda I-\operatorname{adj}\left(\Delta_{D}(\lambda ; \vec{r})\right)\left(A_{0}+\sum_{k=1}^{p_{2}} A_{k} e^{-\lambda v_{k}}\right)\right) .
$$


For $\tilde{\tau} \in(0,1)$, let

$$
\begin{aligned}
q(\mu ; \tilde{\tau}, \vec{r}, \vec{v}) & =\tilde{\tau}^{n} Q\left(\frac{\mu}{\tilde{\tau}} ; \vec{r}, \vec{v}\right) \\
& =\operatorname{det}\left(\mu I-\tilde{\tau} \operatorname{adj}\left(\Delta_{D}\left(\mu ; \frac{\vec{r}}{\tilde{\tau}}\right)\right)\left(A_{0}+\sum_{k=1}^{p_{2}} A_{k} e^{-\mu \frac{v_{k}}{\tilde{\tau}}}\right)\right)
\end{aligned}
$$

Choose $c>0$ arbitrarily. If $q\left(\mu_{0} ; \tilde{\tau}, \vec{r}, \vec{v}\right)=0$ with $\|\vec{r}\|<\tilde{\tau}$ and $\|\vec{v}\|<\tilde{\tau}$, and if $\Re\left(\mu_{0}\right) \geq-c$, then

$$
\left|\mu_{0}\right| \leq \tilde{\tau} f
$$

where

$$
f:=\max _{\|\vec{\rho}\| \leq 1, \Re(\mu) \geq-c}\left\|\operatorname{adj}\left(\Delta_{D}(\mu ; \vec{\rho})\right)\right\|\left(\left\|A_{0}\right\|+\max _{\|\vec{\sigma}\| \leq 1} \sum_{k=1}^{p_{2}}\left\|H_{k}\right\| e^{c \sigma_{k}}\right) .
$$

Hence, if $\|\vec{r}\|<\tilde{\tau}$ and $\|\vec{v}\|<\tilde{\tau}$, then all zeros of $q$ in the half plane

$$
\{\mu \in \mathbb{C}: \Re(\mu) \geq-c\}
$$

lie in the compact set

$$
\mathcal{Q}:=\{\mu \in \mathbb{C}: \Re(\mu) \geq-c \text { and }|\mu| \leq f\}
$$

Next, determine $\hat{\tau}>0$ such that

$$
\begin{aligned}
\min _{\mu \in \partial \mathcal{Q}}\left|\mu^{n}-\operatorname{det}\left(\mu I-\tau \operatorname{adj}\left(\Delta_{D}(\mu ; \vec{\rho})\right)\left(A_{0}+\sum_{k=1}^{p_{2}} A_{k} e^{-\mu \sigma_{k}}\right)\right)\right| & \\
& <\max _{\mu \in \partial \mathcal{Q}}\left|\mu^{n}\right|
\end{aligned}
$$

for all $\tau \in(0, \hat{\tau}]$, for all $\vec{\rho} \in \mathbb{R}^{m}$ and $\vec{\sigma} \in \mathbb{R}^{p_{2}}$ with $\|\vec{\rho}\| \leq 1$ and $\|\vec{\sigma}\| \leq 1$. By Rouché's Theorem it follows that under the condition $\tilde{\tau}<\hat{\tau}, q(\cdot ; \tilde{\tau}, \vec{r}, \vec{v})$ has $n$ zeros in $\mathcal{Q}$ for all $\vec{r} \in\left(\mathbb{R}_{0}^{+}\right)^{m}$ and $\vec{v} \in\left(\mathbb{R}^{+}\right)^{p_{2}}$ with $\|\vec{r}\| \leq \tilde{\tau}$ and $\|\vec{v}\| \leq \tilde{\tau}$. Consequently, $q$ has exactly $n$ zeros in the right half plane $\{\mu \in \mathbb{C}: \Re(\mu) \geq$ $-c\}$, thus, det $\Delta_{N}(; \vec{r}, \vec{v})$ has $n$ zeros in the half plane $\{\lambda \in \mathbb{C}: \Re(\lambda) \geq c / \tilde{\tau}\}$, which implies the statement of the proposition.

\section{Proof of Theorem 5.1}

By Proposition 4.3 the characteristic function $\operatorname{det} \Delta_{N}(\lambda ; \vec{r}, \vec{v})$ has $n$ zeros arbitrarily close to the eigenvalues of the Hurwitz matrix $\left(I+B K_{d}\right)^{-1}(A-$ $\left.B K_{p}\right)$ if $\|\vec{r}\|$ and $\|\vec{v}\|$ are sufficiently small. The remainder of the proof is based on observations on the behavior of the other zeros. Two cases are distinguished:

Case 1: $\operatorname{det} \Delta_{D}(\lambda ; \vec{r}) \not \equiv 1$. 
From a comparison between the statements of Proposition 4.4 and the statements to be proven, it follows that it suffices to prove that $\gamma_{0}<1 \Leftrightarrow \Xi<0$, $\gamma_{0} \geq 1 \Leftrightarrow \Xi>0$ and $\gamma_{0}>1 \Leftrightarrow \bar{C}_{D}\left(\vec{r}_{0}\right)>0$ for some $\vec{r}_{0} \in\left(\mathbb{R}_{0}^{+}\right)^{m}$. This is the case by Proposition 4.2 because for the interdependency structure (21), which satisfies the assumption of Proposition 4.2 by taking $\vec{\beta}=(1, \ldots, 1)$, the quantity $\delta_{0}$ defined in that proposition, reduces to $\gamma_{0}$.

Case 2: $\operatorname{det} \Delta_{D}(\lambda ; \vec{r}) \equiv 1$, or

$$
\operatorname{det}\left(I+\sum_{k=1}^{n_{u}} B E_{k} \sum_{l=1}^{n} K_{d} F_{l} e^{-\lambda\left(\tau_{u_{k}}+\tau_{\dot{x}_{l}}\right)}\right) \equiv 1 .
$$

From Proposition 4.4 robustness of stability can be concluded. In the remainder, it is shown that (39) implies that $\gamma_{0}\left(K_{d}\right) \leq 0$, which is in agreement with the statement of the theorem.

Fix $\vec{\mu} \in[0,2 \pi]^{n_{u}}$ and $\vec{\nu} \in[0,2 \pi]^{n}$. Construct sequences $\left\{d^{(\rho)}\right\}_{\rho \geq 1},\left\{\vec{\tau}_{u}^{(\rho)}\right\}_{\rho \geq 1}$ and $\left\{\vec{\tau}_{x}^{(\rho)}\right\}_{\rho \geq 1}$ with $d^{(\rho)} \in \mathbb{R}, \vec{\tau}_{u}^{(\rho)} \in\left(\mathbb{R}^{+}\right)^{n_{u}}$ and $\vec{\nu}_{x}^{(\rho)} \in\left(\mathbb{R}^{+}\right)^{n}$ for all $\rho$, such that

$$
\begin{array}{ll}
\lim _{\rho \rightarrow \infty} e^{i\left(\mu_{k}+d^{(\rho)} \tau_{u_{k}}^{(\rho)}\right)}=1, & k=1, \ldots, n_{u}, \\
\lim _{\rho \rightarrow \infty} e^{i\left(\nu_{k}+d^{(\rho)} \tau_{\dot{x}_{k}}^{(\rho)}\right)}=1, & k=1, \ldots, n, \\
\lim _{\rho \rightarrow \infty} \tau_{u_{k}}^{(\rho)}=1, & k=1, \ldots, n_{u}, \\
\lim _{\rho \rightarrow \infty} \tau_{\dot{x}_{k}}^{(\rho)}=1, & k=1, \ldots, n .
\end{array}
$$

Note that this is always possible by Kronecker's theorem (Hardy and Wright, 1968, Theorem 444) when taking the components of $\vec{r}^{(\rho)}:=\left(\vec{\tau}_{u}^{(\rho)}, \vec{\tau}_{x}^{(\rho)}\right)$ rationally independent. From

$$
\operatorname{det} \Delta_{D}\left(c+d^{(\rho)} ; \vec{r}^{(\rho)}\right) \equiv 1
$$

it follows that

$$
\operatorname{det}\left(I+e^{-2 c} \sum_{k=1}^{n_{u}} B E_{k} \sum_{l=1}^{n} K_{d} F_{l} e^{i\left(\mu_{k}+\nu_{l}\right)}\right) \equiv 1 .
$$

Hence, the function

$$
c \in \mathbb{R} \rightarrow \operatorname{det}\left(e^{2 c} I+\sum_{k=1}^{n_{u}} B E_{k} \sum_{l=1}^{n} K_{d} F_{l} e^{i\left(\mu_{k}+\nu_{l}\right)}\right)
$$

has no zeros, or equivalently, $-\sum_{k=1}^{n_{u}} B E_{k} \sum_{l=1}^{n} K_{d} F_{l} e^{i\left(\mu_{k}+\nu_{l}\right)}$, has no real strictly positive eigenvalue. As this analysis can be repeated for all $\vec{\mu} \in[0,2 \pi]^{n_{u}}$ and 
$\vec{\nu} \in[0,2 \pi]^{n}:$

$$
\begin{aligned}
\max \{d \in \mathbb{R}: d \in \sigma & \left(-\sum_{k=1}^{n_{u}} B E_{k} \sum_{l=1}^{n} K_{d} F_{l} e^{i\left(\mu_{k}+\nu_{l}\right)}\right) \\
& \text { for some } \left.\vec{\mu} \in[0,2 \pi]^{n_{u}} \text { and } \vec{\nu} \in[0,2 \pi]^{n}\right\} \leq 0 .
\end{aligned}
$$

This result, combined with Lemma 2 of Michiels, et al. (2007), implies that $\gamma_{0}\left(K_{d}\right) \leq 0$.

\section{References}

Abdelaziz, T.H.S and Valášek, M. (2005a),State derivative feedback by lqr for linear time-invariant system, In Proc. of 16th IFAC World Congress

Abdelaziz, T.H.S and Valášek, M. (2005b), Direct Algorithm for Pole Placement by State-derivative Feedback for Multi-inputlinear Systems - Nonsingular Case, Kybernetika, Vol. 41, pp. 637-660

Abdelaziz, T.H.S and Valášek, M. (2005c) Eigenstructure Assignment by Proportional-plus-derivative Feedback for Second-order Linear Control Systems, Kybernetika, Vol. 41, pp. 661-676

Abdelaziz, T.H.S and Valášek, M. (2004), Pole-placement for SISO linear systems by state-derivative feedback, IEEE Proceedings - Control Theory and Applications, Vol 151, pp. 377-385

Avellar, C.E. and Hale, J.H. (1980), On the zeros of exponential polynomials, Mathematical analysis and applications, Vol 73, pp. 434-452

Bellman, R. and Cooke, K.L., (1963), Differential-difference equation, Academic Press, New York

Dyke, S.J., Spencer, B.F., Quast, P., Sain, M.K., Kaspari, D.C. and Soong, T.T., (1996) Acceleration feedback control of MDOF structures, Journal of Engineering Mechanics, Vol. 122,pp. 907-917

Guo, G., Ma, Z. and Qiao, J., (2006), State-PID feedback control with application to a robot vibration absorber, Int. J. of Modelling, Identification and Control, Vol 1, pp. 38-43

Hale, J.K. and Verduyn Lunel, S.M. (2002), Strong stabilization of neutral functional differential equations, IMA Journal of Mathematical Control and Information, Vol. 19, pp. 5-23

Hale, J.K. and Verduyn Lunel, S.M. (2001), Effects of Small Delays on Stability and Control, Operator Theory; Advances and Applicationsl, Vol. 122, pp. 275-301

Hale, J.K. and Verduyn Lunel, S.M., (1993) Introduction to Functional Differential Equations, Applied Math. Sciences, 99, Springer-Verlag, New York

Henry, D. (1974) Linear autonomous neutral functional differential equations, Journal of Differential Equations, Vol. 19, pp. 106-128

Insperger, T., Barton, D.A.W. and Stepan, G. (2008) Criticality of Hopf bi- 
furcation in state-dependent delay model of turning process, International journal of Non-Linear Mechanics, Vol. 43, pp. 140-149

Kautsky, J. and Nichols, N.K., (1985) Robust Pole Assignment in Linear State Feedback, Int. J. Control, Vol. 41, pp. 1129-1155,

Hardy, G. H. and Wright, E. M. (1968) An introduction to the theory of numbers, Oxford University Press

Horowitz, R. , Lib, Y., Oldhama, K., Kona, S. and Huang, X. (2007), Dualstage servo systems and vibration compensation in computer hard disk drives, Control Engineering Practice, Vol. 15, pp. 291305

Leitmann, G. and Reithmeier, E., Robust vibration control of dynamical systems based on the derivative of the state, Archive of Applied Mechanics, Vol. 72 , pp. 856864

Michiels, W. and Niculescu, S.-I. (2007), Stability and stabilization of timedelay systems. An eigenvalue based approach, SIAM, Advances in Design and Control, Vol. 12

Michiels, W., Vyhlídal, T., Zítek, P., Nijmeijer, H. and Henrion, D.,(2007) Strong stability of neutral equations with an arbitrary delay TW Report of the Department of Computer Science, Katholieke Universiteit Leuven, Belgium, No. 498,

Michiels, W. and Vyhlídal, T. (2005) An eigenvalue based approach for the stablization of linear time-delay systems of neutral type, Automatica, Vol. 41, pp. 991-998

Michiels, W., Mondié, S., Roose, D. and Dambrine, M., (2004) The effect of approximating distributed delay control laws on stability",booktitle=" Advances in Time-Delay Systems, Lecture Notes in Computational Science and Engineering, editors Niculescu, S.-I. and Gu, K., Springer Verlag, pp. 207-225

Michiels, W., Engelborghs K., Roose, D. and Dochain D. (2002) Sensitivity to infinitesimal delays in neutral equations, SIAM Journal of Control and Optimization, Vol. 40, pp. 1134-1158

Mondié, S. and Michiels, W., (2003) Finite spectrum assignment of unstable time-delay systems with a safe implementation, IEEE Transactions on Automatic Control, Vol. 48, No. 12, pp.2207-2212

Stepan, G. (2001) Modelling Nonlinear Regenerative Effects in Metal Cutting, Philosophical Transactions of the Royal Society, Vol. 359, pp. 739757

Olgac N., Elmali, H., Hosek, M. and Renzulli, M. (1997) Active vibration control of distributed systems using delayed resonator with acceleration feedback, Transactions of ASME Journal of Dynamic Systems, Measurement and Control, Vol. 119

Valášek, M. and Olgac, N. (1995) Efficient eigenvalue assignments for general linear MIMO systems, Automatica, Vol. 31, pp. 1605-1617

Vyhlídal, T. and Zítek, P. (2003) Quasipolynomial mapping based rootfinder for analysis of Time delay systems, Proc. of IFAC Workshop on Time-Delay Systems TDS'03, Rocquencourt 